Bull. Austral. Math. Soc.

VoL. $72(2005)$ [455-459]

\title{
A FUNCTIONAL INEQUALITY FOR THE POLYGAMMA FUNCTIONS
}

Horst Alzer

Let

$$
\Delta_{n}(x)=\frac{x^{n+1}}{n !}\left|\psi^{(n)}(x)\right| \quad(x>0 ; n \in \mathrm{N}),
$$

where $\psi$ denotes the logarithmic derivative of Euler's gamma function. We prove that the functional inequality

$$
\Delta_{n}(x)+\Delta_{n}(y)<1+\Delta_{n}(z), \quad x^{r}+y^{r}=z^{r},
$$

holds if and only if $0<r \leqslant 1$. And, we show that the converse is valid if and only if $r<0$ or $r \geqslant n+1$.

\section{INTRODUCTION}

In 1973, Grünbaum [6] presented the following elegant inequality for the Bessel function $J_{0}$.

$$
J_{0}(x)+J_{0}(y) \leqslant 1+J_{0}(z), \quad x^{2}+y^{2}=z^{2} .
$$

Askey [4] offered a new proof of (1.1) and showed that (1.1) can be extended to $J_{\alpha}$ with $\alpha>0$.

$$
J_{\alpha}^{*}(x)+J_{\alpha}^{*}(y) \leqslant 1+J_{\alpha}^{*}(z), \quad x^{2}+y^{2}=z^{2},
$$

where

$$
J_{\alpha}^{*}(x)=2^{\alpha} \Gamma(\alpha+1) x^{-\alpha} J_{\alpha}(x) .
$$

It is natural to ask whether there exist other special functions which satisfy inequalities of Grünbaum-type.

The logarithmic derivative of the gamma function, $\psi=\Gamma^{\prime} / \Gamma$, is known in the literature as the digamma or psi function. Its derivatives

$$
\psi^{\prime}, \psi^{\prime \prime}, \psi^{\prime \prime \prime}, \ldots
$$

Received 25th July, 2005

I thank the referee for helpful comments.

Copyright Clearance Centre, Inc. Serial-fee code: 0004-9727/05 \$A2.00+0.00. 
are called polygamma functions. We have the integral and series representations

$$
\begin{aligned}
\psi^{(n)}(x) & =(-1)^{n+1} \int_{0}^{\infty} e^{-x t} \frac{t^{n}}{1-e^{-t}} d t \\
& =(-1)^{n+1} n ! \sum_{k=0}^{\infty} \frac{1}{(x+k)^{n+1}} \quad(x>0 ; n \in \mathrm{N}) .
\end{aligned}
$$

These functions have interesting applications in various fields. In particular, they play an important role in mathematical physics. Their main properties can be found, for instance, in [1, Chapter 6]. Inequalities for digamma and polygamma functions are discussed in [3]. We also refer to [5], where a survey on gamma function inequalities is given.

In this note, we show that the trigamma function $\psi^{\prime}$ satisfies

$$
1+z^{2} \psi^{\prime}(z)<x^{2} \psi^{\prime}(x)+y^{2} \psi^{\prime}(y), \quad x^{2}+y^{2}=z^{2}
$$

Actually, (1.3) is a special case of a more general inequality involving the function

$$
\Delta_{n}(x)=\frac{x^{n+1}}{n !}\left|\psi^{(n)}(x)\right| \quad(x>0 ; n \in \mathrm{N}),
$$

which we provide in the next section.

\section{MAIN RESUlt}

To prove our theorem we need properties of $\Delta_{n}$ and its derivative.

LEMMA . Let $n \geqslant 1$ be an integer. The functions $\Delta_{n}$ and $\Delta_{n}^{\prime}$ are strictly increasing on $(0, \infty)$. Moreover,

$$
\lim _{x \rightarrow 0} \Delta_{n}(x)=1 \text { and } \lim _{x \rightarrow 0} \Delta_{n}^{\prime}(x)=0
$$

ProOF: The monotonicity and the convexity of $\Delta_{n}$ are proved in [2] and [3], respectively. Using the recurrence formula

$$
\left|\psi^{(n)}(x)\right|=\left|\psi^{(n)}(x+1)\right|+\frac{n !}{x^{n+1}}
$$

(see $[1$, p. 260]), we obtain

$$
\Delta_{n}(x)=1+\frac{x^{n+1}}{n !}\left|\psi^{(n)}(x+1)\right|
$$

and

$$
\Delta_{n}^{\prime}(x)=\frac{n+1}{n !} x^{n}\left|\psi^{(n)}(x+1)\right|-\frac{x^{n+1}}{n !}\left|\psi^{(n+1)}(x+1)\right|
$$

From (2.2) and (2.3) we conclude that (2.1) holds. 
We are now in a position to prove (1.3) and its extension to higher derivatives.

TheOREM. Let $n \geqslant 1$ be an integer and let $r \neq 0$ be a real number. The inequality

$$
\Delta_{n}(x)+\Delta_{n}(y)<1+\Delta_{n}(z)
$$

holds for all positive real numbers $x, y, z$ with $x^{r}+y^{r}=z^{r}$ if and only if $0<r \leqslant 1$. And,

$$
1+\Delta_{n}(z)<\Delta_{n}(x)+\Delta_{n}(y)
$$

is valid for all $x, y, z>0$ with $x^{r}+y^{r}=z^{r}$ if and only if $r<0$ or $r \geqslant n+1$.

ProOF: We define for $x, y>0$ :

$$
f_{n, r}(x, y)=1+\Delta_{n}\left(\left(x^{r}+y^{r}\right)^{1 / r}\right)-\Delta_{n}(x)-\Delta_{n}(y)
$$

First, we assume that $f_{n, r}(x, y)>0$ for all $x, y>0$. Then we obtain

$$
f_{n, r}(x, x)=1+\Delta_{n}\left(2^{1 / r} x\right)-2 \Delta_{n}(x)>0 .
$$

The asymptotic formula

$$
\left|\psi^{(n)}(x)\right| \sim \frac{(n-1) !}{x^{n}}+\frac{n !}{2 x^{n+1}}+\cdots \quad(x \rightarrow \infty)
$$

(see $[1$, p. 260]), gives

$$
\lim _{x \rightarrow \infty} \frac{\Delta_{n}(x)}{x}=\frac{1}{n}
$$

Thus,

$$
0 \leqslant \lim _{x \rightarrow \infty} \frac{f_{n, r}(x, x)}{x}=\frac{1}{n}\left(2^{1 / r}-2\right) .
$$

This leads to $0<r \leqslant 1$.

Next, we prove that if $0<r \leqslant 1$, then

$$
f_{n, r}(x, y)>0 \text { for all } x, y>0 .
$$

Since $r \mapsto\left(x^{r}+y^{r}\right)^{1 / r}$ is decreasing on $(0, \infty)$, we conclude from the Lemma that $r \mapsto f_{n, r}(x, y)$ is also decreasing on $(0, \infty)$. Hence,

$$
f_{n, r}(x, y) \geqslant f_{n, 1}(x, y)=1+\Delta_{n}(x+y)-\Delta_{n}(x)-\Delta_{n}(y)=g_{n}(x, y), \quad \text { say. }
$$

Applying the Lemma again we obtain

$$
\frac{\partial}{\partial x} g_{n}(x, y)=\Delta_{n}^{\prime}(x+y)-\Delta_{n}^{\prime}(x)>0
$$

This leads to

$$
g_{n}(x, y)>g_{n}(0, y)=0
$$


From (2.7) and (2.8) it follows that (2.6) holds.

Now, we consider (2.5). Let $r>0$. We suppose that

$$
f_{n, r}(x, y)<0=f_{n, r}(0, y) \quad(x, y>0) .
$$

Partial differentiation gives

$$
\frac{1}{x^{n}} \frac{\partial}{\partial x} f_{n, r}(x, y)=x^{r-1-n} \Delta_{n}^{\prime}\left(\left(x^{r}+y^{r}\right)^{1 / r}\right)\left(x^{r}+y^{r}\right)^{1 / r-1}-\frac{\Delta_{n}^{\prime}(x)}{x^{n}}
$$

Formula (2.3) yields

$$
\lim _{x \rightarrow 0} \frac{\Delta_{n}^{\prime}(x)}{x^{n}}=\frac{n+1}{n !}\left|\psi^{(n)}(1)\right|
$$

and an application of the Lemma implies

$$
\lim _{x \rightarrow 0} \Delta_{n}^{\prime}\left(\left(x^{r}+y^{r}\right)^{1 / r}\right)\left(x^{r}+y^{r}\right)^{1 / r-1}=\Delta_{n}^{\prime}(y) y^{1-r}>0 .
$$

From (2.9)-(2.12) we conclude that $r-1-n \geqslant 0$.

It remains to show that if $r<0$ or $r \geqslant n+1$, then

$$
f_{n, r}(x, y)<0 \text { for all } x, y>0
$$

Let $r<0$. We have

$$
\left(x^{r}+y^{r}\right)^{1 / r}<\min (x, y)
$$

so that the Lemma implies

$$
f_{n, r}(x, y)<1+\Delta_{n}(\min (x, y))-\Delta_{n}(x)-\Delta_{n}(y)<0 .
$$

Let $r \geqslant n+1$ and

$$
s=s_{n}(x, y)=\left(x^{n+1}+y^{n+1}\right)^{1 /(n+1)} .
$$

We obtain

$$
f_{n, r}(x, y) \leqslant 1+\Delta_{n}(s)-\Delta_{n}(x)-\Delta_{n}(y)=u_{n}(x, y), \quad \text { say. }
$$

Differentiation yields

$$
\frac{\partial}{\partial x} u_{n}(x, y)=x^{n}\left[v_{n}(s)-v_{n}(x)\right]
$$

where

$$
v_{n}(x)=\frac{\Delta_{n}^{\prime}(x)}{x^{n}}
$$

Using

$$
\frac{1}{x}=\int_{0}^{\infty} e^{-x t} d t \quad(x>0)
$$


the integral representation (1.2), and the convolution theorem for Laplace transforms, we obtain

$$
n ! \frac{v_{n}^{\prime}(x)}{x}=-\frac{n+2}{x}\left|\psi^{(n+1)}(x)\right|+\left|\psi^{(n+2)}(x)\right|=\int_{0}^{\infty} e^{-x t} Z_{n}(t) d t
$$

where

$$
Z_{n}(t)=\frac{t^{n+2}}{1-e^{-t}}-(n+2) \int_{0}^{t} \frac{s^{n+1}}{1-e^{-s}} d s
$$

We have

$$
Z_{n}(0)=0 \text { and } Z_{n}^{\prime}(t)=-\frac{t^{n+2} e^{-t}}{\left(1-e^{-t}\right)^{2}}
$$

This implies that $Z_{n}$ is negative on $(0, \infty)$. From $(2.16)$ we find that $v_{n}$ is strictly decreasing on $(0, \infty)$. Since $s>x$, we obtain from (2.15) that

$$
u_{n}(x, y)<u_{n}(0, y)=0
$$

Combining (2.14) and (2.17) we conclude that (2.13) is valid.

\section{REFERENCES}

[1] M. Abramowitz and I.A. Stegun (eds.), Handbook of mathematical functions with formulas and mathematical tables (Dover Publications Inc., New York, 1965).

[2] H. Alzer, 'Mean-value inequalities for the polygamma functions', Aequationes Math. 61 (2001), 151-161.

[3] H. Alzer, 'Sharp inequalities for the digamma and polygamma functions', Forum Math. 16 (2004), 181-221.

[4] R. Askey, 'Grünbaum's inequality for Bessel functions', J. Math. Anal. Appl. 41 (1973), 122-124.

[5] W. Gautschi, 'The incomplete gamma function since Tricomi', in Tricomi's ideas and contemporary applied mathematics, Atti Convegni Lincei 147 (Accad. Naz. Lincei, Rome, 1998), pp. 203-237.

[6] F.A. Grünbaum, 'A new kind of inequality for Bessel functions', J. Math. Anal. Appl. 41 (1973), 115-121.

Morsbacher Str. 10

D-51545 Waldbröl

Germany

e-mail alzerhorst@freenet.de 